\title{
Network Analysis Reveals Increased Integration during Emotional and Motivational Processing
}

\author{
Joshua Kinnison, Srikanth Padmala, Jong-Moon Choi, and Luiz Pessoa \\ Department of Psychology, University of Maryland, College Park, Maryland 20742
}

In recent years, a large number of human studies have investigated large-scale network properties of the brain, typically during the resting state. A critical gap in the knowledge base concerns the understanding of network properties of a focused set of brain regions during task conditions engaging these regions. Although emotion and motivation recruit many brain regions, it is currently unknown how they affect network-level properties of inter-region interactions. In the present study, we sought to characterize network structure during "mini-states" engendered by emotional and motivational cues investigated in separate studies. To do so, we used graph-theoretic network analysis to probe network-, community-, and node-level properties of the trial-by-trial functional connectivity between regions of interest. We used methods that operate on weighted graphs that make use of the continuous information of connectivity strength. In both the emotion and motivation datasets, global efficiency increased and decomposability decreased. Thus, processing became less segregated with the context signaled by the cue (potential shock or potential reward). Our findings also revealed several important features of inter-community communication, including notable contributions of the bed nucleus of the stria terminalis, anterior insula, and thalamus during threat and of the caudate and nucleus accumbens during reward. Together, the results suggest that one way in which emotional and motivational processing affect brain responses is by enhancing signal communication between regions, especially between cortical and subcortical ones.

\section{Introduction}

The past years have witnessed an explosion in the investigations of how brain regions are organized into networks. Network analysis of neuroimaging data has focused almost exclusively on characterizing the large-scale properties of resting-state datasets in which participants lay passively in the scanner without performing an explicit task (Bullmore and Sporns, 2009; Wang et al., 2010). However, a critical gap in the knowledge base concerns the understanding of network properties of a focused set of brain regions during task conditions engaging these regions.

In the present study, we sought to characterize network structure during "mini-states" engendered by emotional and motivational cues. Previous studies have identified a number of regions involved in threat processing, including dorsomedial prefrontal cortex (PFC), anterior insula, bed nucleus of the stria terminalis (BNST), and thalamus (Dalton et al., 2005; Chandrasekhar et al., 2008; Mobbs et al., 2010; Somerville et al., 2010; Choi et al., 2012). Reward processing engages, among others, dorsomedial PFC and ventral/dorsal striatum (Savine and Braver, 2010; Aarts et al., 2011; Padmala and Pessoa, 2011). Little is known, however, about how threat and reward influence network properties of

Received Feb. 20, 2012; revised April 5, 2012; accepted April 27, 2012.

Author contributions:S.P., J.-M.C., and L.P. designed research; S.P. and J.-M.C. performed research; J.K. analyzed data; J.K., S.P., and L.P. wrote the paper.

This work was supported by National Institute of Mental Health Grant 1R01 MH071589 (L.P.). We thank Philip Spechler for assistance with preparation of this manuscript.

Correspondence should be addressed to Luiz Pessoa, Department of Psychology, University of Maryland, College Park, MD 20742.E-mail: pessoa@umd.edu.

DOI:10.1523/JNEUROSCI.0821-12.2012

Copyright $\odot 2012$ the authors $\quad 0270-6474 / 12 / 328361-12 \$ 15.00 / 0$ functional interactions across the many brain regions recruited by these manipulations.

To address this question, two distinct paradigms were investigated in which emotional/motivational cues preceded the execution of a response-conflict task (see Fig. 1). In the emotion task, participants viewed an initial cue that indicated whether they were in a threat or safe trial, whereas in the motivation task, participants viewed an initial cue that indicated whether they were in a reward or control trial. Here, we used graph-theoretic measures to characterize emotional and motivational processing. Accordingly, our analyses focused on responses generated to the cues themselves and not on the cognitive task that was performed later in the trial.

It has been proposed that increases in functional connectivity can be interpreted as evidence for increased functional integration (Friston et al., 1997). Whereas changes in functional connectivity have been reported many times in the emotion and motivation literatures (Pessoa et al., 2002; Harsay et al., 2011), previous studies have not investigated how functional connectivity is potentially altered simultaneously across all regions engaged by emotional/motivational stimuli. We were particularly interested in evaluating the roles of key brain regions, such as the BNST and anterior insula during threat and the caudate and nucleus accumbens during reward. Because the analyses used here consider the entire correlation matrix (i.e., the pairwise functional connectivity between every pair of regions), they are able to probe general properties of information segregation and integration (Bullmore and Sporns, 2009). Specifically, we applied a community-detection algorithm to the set of regions robustly engaged by threat and, separately, reward and compared, among 
A Emotion study

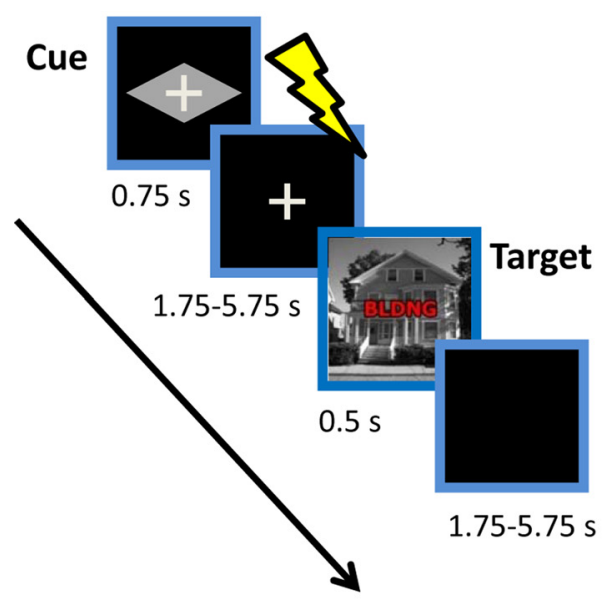

B
Motivation study

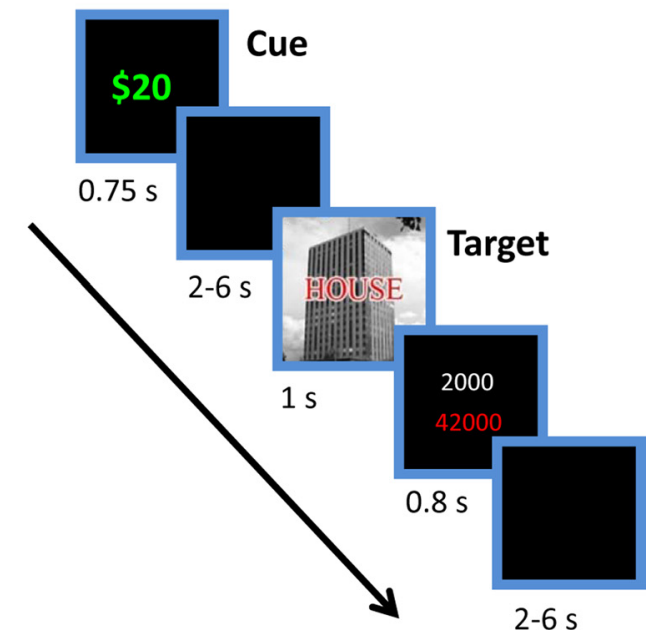

Figure 1. Experimental paradigms. In both tasks, an initial cue signaled the trial condition: safe and threat in the emotion study, and control and reward in the motivation study. A, Subjects performed a response- conflict task under two contexts, safe and threat. During the threat condition (shown here), a cue stimulus (diamond) signaled that a mild electric shock could occur during the delay period after cue offset and before the target display (independently of task performance). During the subsequent target phase, participants were asked to indicate whether the picture contained a house or a building, while ignoring the superimposed word. During the safe condition (data not shown), the trial structure was identical, except for the shape of the cue stimulus (rectangle) and the fact that shocks were never administered during the delay period. B, Subjects performed a response- conflict task under two contexts, control and reward. During the reward condition (shown here), a cue stimulus ("\$20") signaled that participants would be rewarded for fast and correct performance; during the control condition (not shown here), a cue stimulus ("\$00") signaled that no reward was involved. During the subsequent target phase, participants were asked to indicate whether the picture contained a house or a building, while ignoring the superimposed word. After the target stimulus, subjects were informed about the potential reward and about the total points accrued.

others, global efficiency and decomposability measures that have been used across many applications of network analysis (Newman, 2010).

\section{Materials and Methods}

\section{Emotion dataset}

For additional details, see Choi et al. (2012).

Subjects. The study was approved by the Institutional Review Board of Indiana University (Bloomington, IN). Data from 41 participants $(21 \pm$ 2.40 years old; 22 females) were used who were free from psychological and neurological conditions as measured via self-report. Participants were right-handed, had normal or corrected-to-normal vision, and gave informed written consent.

Stimuli and behavioral paradigm. Each trial started with the presentation of a rectangle- or diamond-shaped cue stimulus $(750 \mathrm{~ms})$ that indicated the experimental condition (safe, threat), followed by a $1.75-5.75 \mathrm{~s}$ variable delay period. The threat cue, which was counterbalanced across participants, indicated that a mild electric shock could be delivered during the delay period (independently of task performance). To calibrate the intensity of the electric shock, each participant was asked to choose his/her own stimulation level immediately before functional imaging, such that the stimulus would be "highly unpleasant but not painful." After each run, participants were asked about the unpleasantness of the shock and were asked to, if needed, recalibrate it. Shocks were administered with an electrical stimulator (Coulbourn Instruments) on the fourth ("ring") and fifth ("pinky") fingers of the nondominant left hand. During the threat condition, physical shocks were administered on 33\% of the trials (participants were not informed about the probability of shock). Skin conductance response data were collected using the MP-150 system (BIOPAC Systems) at a sampling rate of $250 \mathrm{~Hz}$ by using MRIcompatible electrodes attached to the index and middle fingers of the left hand.

After the delay, the target display was presented for $500 \mathrm{~ms}$, followed by a $1.75-5.75 \mathrm{~s}$ variable intertrial interval (ITI). During the target phase, participants performed a response-conflict task (Fig. 1A). Both delay and ITI durations were selected from an exponential distribution favoring shorter intervals and helped in the robust estimation of separate cueand target-related responses.
Each participant performed six "runs" of the main task (seven runs for one participant). Each run consisted of 54 trials, resulting in a total of 324 trials. Because the current study focused exclusively on the cue phase, we pooled the cue phase data from all trials (independent of target phase congruency) for each condition (safe, threat). After deletion of the actual physical shock trials and the subsequent safe trials, there were a total of 108 trials per cue condition.

MR data acquisition. MR data were collected using a 3 tesla Siemens TRIO scanner (Siemens Medical Systems) with a 32-channel head coil (without parallel imaging). Each scanning session began with a highresolution MPRAGE anatomical scan (TR, $1900 \mathrm{~ms}$; TE, $4.15 \mathrm{~ms}$; TI, $1100 \mathrm{~ms} ; 1 \mathrm{~mm}$ isotropic voxels; $256 \mathrm{~mm}$ field of view). Subsequently, in each functional run of the main experiment, 169 EPI volumes were acquired with a TR of $2500 \mathrm{~ms}$ and TE of $25 \mathrm{~ms}$. Each volume consisted of 44 oblique slices with a thickness of $3 \mathrm{~mm}$ and an in-plane resolution of $3 \times 3 \mathrm{~mm}$ ( $192 \mathrm{~mm}$ field of view). Slices were positioned $\sim 30^{\circ}$ relative to the plane defined by the line connecting the anterior and posterior commissures to reduce susceptibility effects in regions such as the amygdala.

$f M R I$ data analysis. Preprocessing of the data was done using tools from the AFNI software package [http://afni.nimh.nih.gov/afni (Cox, 1996)], and standard procedures were used (Choi et al., 2012). Cuerelated responses were estimated starting from event onset to $15 \mathrm{~s}$ after onset using cubic spline basis functions (thus, no shape assumptions were made). As an index of cue activation, we averaged the estimated responses at 5 and $7.5 \mathrm{~s}$ after stimulus onset (as determined via the spline-based estimates) for the safe and threat conditions, separately. Note that correlations between cue and target regressors were modest $(\leq 0.34)$, allowing us to separately estimate cue and target phase responses. Because the cue was always followed by the delay period, no attempt was made to separate cue responses from those during the delay period. Thus, the responses estimated with respect to cue onset combined these two components.

Regions of interest definitions. Regions of interest (ROIs) were based on 5 -mm-radius spheres centered on peak voxels of the contrast threat versus safe during the cue phase defined at the group level.

Estimation of trial-by-trial responses at cue phase. Cue-related responses were estimated on a trial-by-trial basis via the method described previously (Rissman et al., 2004). For each participant, a design matrix 
was set up such that the cue phase of each correct trial was modeled as a separate event, and the target phase of those trials was modeled in a standard way using a single regressor for all the trials of each congruency condition. Response estimates for the cue phase of each trial, as well as for the target phase for each condition, were obtained by convolving regressors with the canonical hemodynamic response (Cohen, 1997). Actual physical shock trials, the subsequent safe trials, and error trials were modeled using additional regressors of no interest. Importantly, correlations between any single-trial cue regressor and target phase regressors was low $(r<0.25)$, thus providing meaningful estimates for single trials. However, it should be stated that, for some individual trials, some "bleed-over" may have occurred. For an evaluation of this method in the context of functional connectivity analysis, see Zhou et al. (2009). For applications of this technique in regular connectivity analysis, see Padmala and Pessoa (2011) and Choi et al. (2012).

\section{Motivation dataset}

For additional details, see Padmala and Pessoa (2011).

Subjects. The study was approved by the Institutional Review Board of Indiana University. Data from 50 participants $(22 \pm 5$ years old; 28 females) were used who were free from psychological and neurological conditions as measured via self-report. Participants were right-handed, had normal or corrected-to-normal vision, and gave informed written consent.

Stimuli and behavioral paradigm. Each trial began with a cue indicating the motivation condition (" $\$ 00$ " or “ $\$ 20$ ") shown for $750 \mathrm{~ms}$, followed by a $2-6 \mathrm{~s}$ variable delay period. The reward cue indicated that participants had the potential to earn extra money based on fast and accurate performance (totaling \$20). After the delay, the target display was presented for $1000 \mathrm{~ms}$, during which participants performed a responseconflict task (Fig. 1B). After $200 \mathrm{~ms}$ from the offset of the target (not shown in Fig. $1 B$ ), participants received visual feedback for $800 \mathrm{~ms}$ consisting of the total number of points won on the trial, as well as their cumulative earnings (in points) until that moment in time. Finally, a 2-6 s ITI containing a blank screen terminated the trial. Both interstimulus interval and ITI were selected from an exponential distribution favoring shorter intervals and helped in the robust estimation of separate cue- and target-related responses.

Each participant performed six runs of the conflict task. Each run consisted of 36 trials, resulting in a total of 216 trials. As in the emotion dataset, because the analysis focused exclusively on the cue phase, we pooled the cue phase data from all trials (independent of target phase congruency) for each cue condition (control, reward). A total of 108 trials per cue condition were used.

MR data acquisition. Parameters were the same as in the emotion dataset, with the exception that each main run was collected with 165 volumes.

MRI data analysis. Data analysis followed the same steps as in the emotion dataset. Again, correlations between cue and target regressors were modest $(\leq 0.37)$, allowing us to separately estimate cue and target phase responses. Because the cue was always followed by the delay period, again, no attempt was made to separate cue responses from those during the delay period.

ROI definitions. ROIs were based on 5-mm-radius spheres centered on the peak voxels of the contrast reward versus control during the cue phase at the group level.

Estimation of trial-by-trial responses at cue phase. Data analysis followed the same procedures used in the emotion dataset. Again, correlations between any single-trial cue regressor and target phase regressors was low $(r<0.20)$, thus providing meaningful estimate for single trials.

\section{Network construction}

Individual level. Networks were constructed first at the participant level, with two networks per participant. In what we refer to as the emotion dataset, safe and threat networks were constructed. In what we refer to as the motivation dataset, control and reward networks were constructed. In the emotion dataset, nodes corresponded to ROIs defined via the contrast of threat $>$ safe, as defined in our previous study (Choi et al., 2012). A total of 16 ROIs were used (Fig. 2A, Table 1), 13 of which were exactly as reported previously. The three regions added were subcortical counterparts to those observed in the right hemisphere, specifically, left thalamus, left BNST/caudate, and left basal forebrain. Although these did not survive the thresholding used in the previous report, they survived at $p<0.001$, uncorrected for multiple comparisons, and were included here so that bilateral subcortical regions could be considered.

In the motivation dataset, nodes corresponded to ROIs defined via the contrast of reward $>$ control, as defined in our previous study (Padmala and Pessoa, 2011). A total of 22 ROIs were used (Fig. 2B, Table 2), which corresponded to the regions defined in our previous study with the exception of the visual regions, which were not used here to keep the emotion and motivation networks of similar size and given the present goal of characterizing the interactions between frontoparietal regions and subcortical ones.

For each dataset, two connectivity matrices were defined based on the functional connectivity between all pairs of regions (emotion dataset: safe and threat; motivation dataset: control and reward). Functional connectivity was determined based on trial-based cue phase responses from all pairs of regions. Whereas regular Pearson's correlation is frequently used as a measure of functional connectivity in network analysis of fMRI data, here we used a robust measure of connectivity to mitigate the potential impact of "extreme" points that frequently distort nonrobust measures of linear association. To do so, we used robust regression by using reweighted least squares with a bisquare weighting function (Street et al., 1988) available in MATLAB (MathWorks) as the function robustfit. Specifically, we calculated Pearson's $r(X, Y)$ product-moment correlation using the reweighted trial-based estimates to determine the $N \times N$ connectivity matrix, where $N$ was the number of ROIs in the given network:

$$
\begin{gathered}
b=\operatorname{cov}\left(X_{\text {ReWeighted }}, Y_{\text {Reweighted }}\right) / \operatorname{var}\left(X_{\text {ReWeighted }}\right), \\
r(X, Y)=b \times \operatorname{SD}\left(X_{\text {Reweighted }}\right) / \mathrm{SD}\left(Y_{\text {ReWeighted }}\right),
\end{gathered}
$$

where cov, var, and SD, are covariance, variance, and standard deviation, respectively.

Finally, negative edge weights (i.e., negative correlations) were set to zero. Because many network measures are incapable of handling negative edge weights, a common practice is to consider the positive and negative weights separately (Schwarz and McGonigle, 2011) or ignore negative correlations entirely (Rubinov and Sporns, 2010). In the present case, we found no evidence of meaningful negative correlations. Over all subjectlevel networks, only a median of $0.4 \%$ of edges were negative. Importantly, at the group-level networks (see below), no negative weights were present.

Group level. To generate a group-level network, individual data were collapsed into a median correlation matrix. This was done before removing negative weights. At the group level, there were thus four networks: safe and threat (emotion dataset), as well as control and reward (motivation dataset).

\section{Network analysis}

Data analysis was performed in MATLAB. Global efficiency measures the average strength of the shortest paths in the network and can be interpreted as the overall "efficiency of communication." Minimizing the cost of communication over the most direct paths in the network affords more efficient information transfer throughout the network. Global efficiency requires as input a measure of node dissimilarity, or the "cost" of a connection, which was defined as the inverse of the functional connection weight (i.e., $1 / w$ ). Note that a value of zero does not pose problems in this respect because in such cases the algorithm is designed to handle the exception (Brain Connectivity Toolbox developed by O. Sporns, Indiana University, Bloomington, IN). More formally, global efficiency $E$ is defined as follows:

$$
E=\frac{1}{n(n-1)} \sum_{\substack{i, j \in V \\ i \neq j}} \frac{1}{d_{i j}},
$$



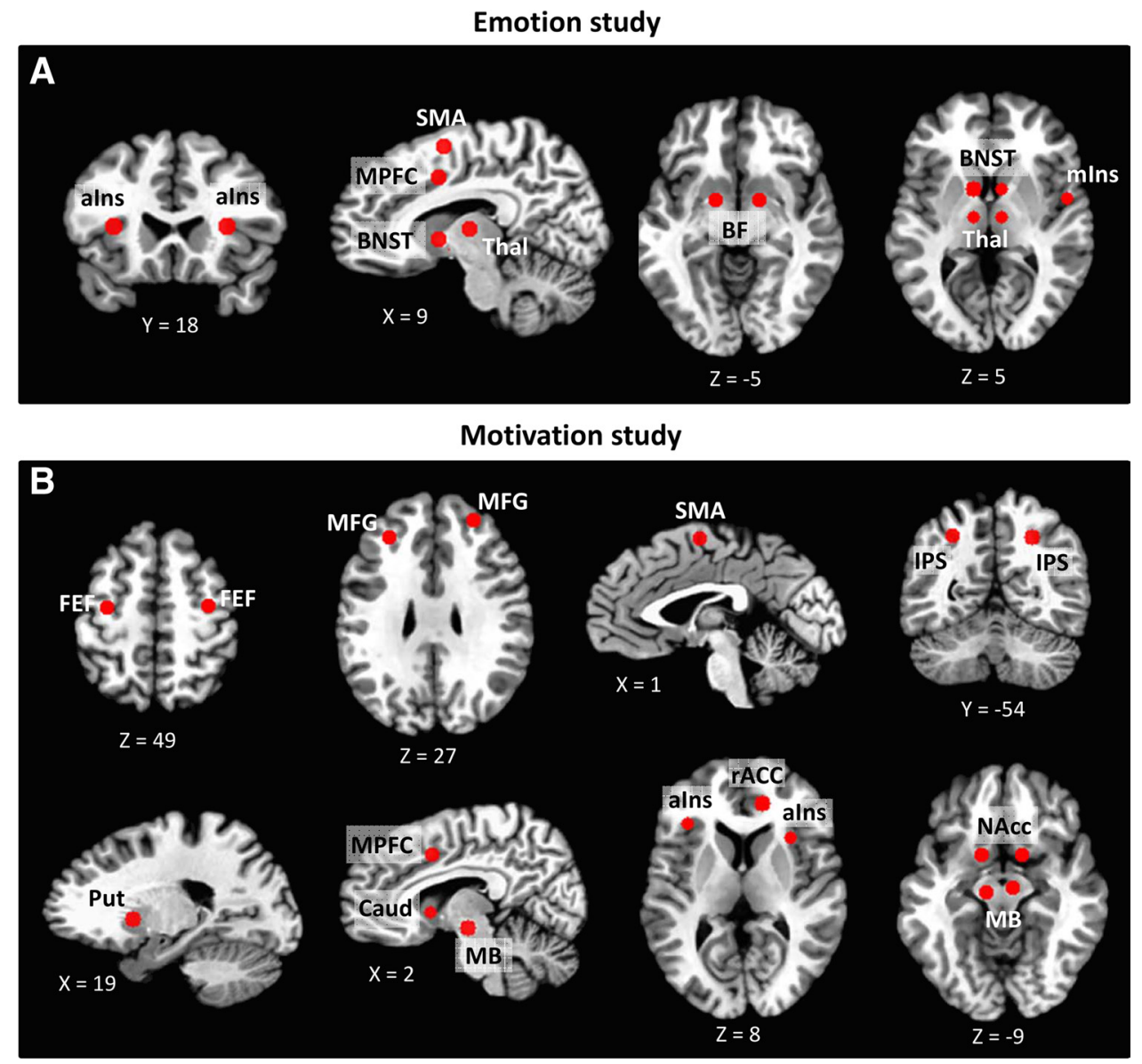

Figure 2. A, Anatomical slices showing ROIs used in the network analysis of the emotion dataset. For abbreviations, see Table 1. B, Anatomical slices showing Rols used in the network analysis of the motivation dataset. For abbreviations, see Table 2.

where $n$ is the number of nodes in the network, $V$ is the set of nodes, and $d_{i j}$ is the cost of the shortest path between nodes $i$ and $j$.

Researchers in network theory have proposed a measure they call "modularity" to characterize the extent to which "like is connected to like" in a network (Newman, 2010). In a nutshell, it indicates the extent to which networks can be decomposed into subnetworks. Unfortunately, the term modularity has many unintended connotations in cognitive science. Because of that, we have chosen to discuss our results in terms of "decomposability." However, because modularity is a technical term in network theory, to avoid confusion, we retain this term in the formal description provided here in Materials and Methods.

For weighted networks, the modularity $Q$ of a given set of community assignments for a graph is a measure of the total weight of connections found within the assigned modules versus the total weight of connections predicted in a random graph with equivalent degree distribution. A positive $Q$ indicates that the number of intramodule connections exceeds those predicted statistically. Thus, modularity optimization returns the set of node assignments that provide the highest $Q$, that is, the optimal "modular" decomposition of the network (i.e., graph). More formally, modularity $Q$ was defined as follows:

$$
Q\left(P_{k}\right)=\sum_{c=1}^{k}\left[\frac{A\left(V_{c}, V_{c}\right)}{A(V, V)}-\left(\frac{A\left(V_{c}^{\prime}, V^{\prime \prime}\right.}{A(V, V)}\right)^{2}\right],
$$

where $V$ is the set of all nodes, $P_{k}$ is a partitioning of the network into $k$ communities, $V_{c}$ is the set of nodes within community $c, w(i, j)$ is the weight of connection between nodes $i$ and $j$, and $A\left(V^{\prime}, V^{\prime \prime}\right)=\sum_{i \in V^{\prime}, j \in V^{\prime \prime}} W(i, j)$ is the sum of all edges between node set $V^{\prime}$ and node set $V^{\prime \prime}$ (for additional discussion, see White and Smyth, 2005). Community detection was performed via Clauset-Newman-Moore modularity optimization (Clauset et al., 2004) on the group-level network. Briefly, community detection determines a partition of a network into subnetworks, such that each node is assigned to one community, and $Q$ assumes a maximal value.

Whereas community detection is many times applied to sizeable networks (say, $>100$ nodes), many standard benchmarks use smaller networks, such as Zachary's network of karate club members with 34 nodes (Zachary, 1977), comparable in size with the networks investigated here. Nevertheless, we further evaluated the quality of the community structure detected in the two datasets. According to the definition of network modularity, a graph has community structure with respect to a random graph of equal size and expected degree distribution. Thus, the modularity maximum of a graph reveals a significant community structure only if it is appreciably larger than the modularity maximum of random graphs of the same size and expected degree distribution (Fortunato, 2010). The significance of the modularity maximum $Q_{\mathrm{MAX}}$ for a graph can be estimated by calculating the maximum modularity for many realizations of the null model obtained from the original graph by randomly rewiring its edges. The statistical significance of $Q_{\mathrm{MAX}}$ is indicated by the distance of $Q_{\mathrm{MAX}}$ from the null model average $\left\langle Q_{\mathrm{NM}}\right\rangle$ in units of the $\mathrm{SD} \sigma_{\mathrm{NM}}$, i.e., by the $z$-score:

$$
z=\frac{Q_{\mathrm{MAX}}-\left(Q_{\mathrm{NM}}\right)}{\sigma_{\mathrm{NM}}} .
$$

If $z$ is substantially $>1, Q_{\mathrm{MAX}}$ indicates strong community structure, and cutoff values of $2-3$ are customary (Fortunato, 2010). Here, we evaluated community structure by using the equation above and by using 100 network randomizations via a recently suggested method (Rubinov and Sporns, 2011).

To improve the robustness of community assignments, community detection was done at the group-level network. However, it was impor- 
Table 1. ROI peak locations for emotion study (Talairach coordinates)

\begin{tabular}{|c|c|c|c|c|}
\hline \multirow[b]{2}{*}{ Location } & \multicolumn{4}{|c|}{ Threat $>$ safe } \\
\hline & $x$ & $y$ & $z$ & Label \\
\hline \multicolumn{5}{|c|}{ Parietal/temporal } \\
\hline \multicolumn{5}{|c|}{ Inferior parietal gyrus/superior temporal gyrus } \\
\hline $\mathrm{L}$ & -58 & -25 & 23 & IPG_L \\
\hline R & 62 & -37 & 23 & IPG_R \\
\hline \multicolumn{5}{|l|}{ Frontal } \\
\hline \multicolumn{5}{|c|}{ Posterior inferior frontal gyrus } \\
\hline L & -58 & 5 & 11 & pIFG_L \\
\hline \multicolumn{5}{|c|}{ Posterior inferior frontal gyrus/mid-insula } \\
\hline R & 50 & -1 & 8 & pIFG_R \\
\hline \multicolumn{5}{|c|}{ Supplementary motor area } \\
\hline R & 8 & 2 & 56 & SMA_R \\
\hline \multicolumn{5}{|c|}{ Mid-insula } \\
\hline $\mathrm{R}$ & 32 & 5 & 11 & mlns_R \\
\hline \multicolumn{5}{|c|}{ Medial prefrontal cortex } \\
\hline $\mathrm{L}$ & -4 & 5 & 32 & MPFC_L \\
\hline $\mathrm{R}$ & 11 & 5 & 38 & MPFC_R \\
\hline \multicolumn{5}{|c|}{ Anterior insula } \\
\hline L & -31 & 17 & 11 & alns_L \\
\hline $\mathrm{R}$ & 29 & 20 & 11 & alns_R \\
\hline \multicolumn{5}{|c|}{ Subcortical } \\
\hline \multicolumn{5}{|c|}{ Thalamus } \\
\hline $\mathrm{R}$ & 8 & -13 & 8 & Thal_R \\
\hline $\mathrm{L}$ & -10 & -13 & 8 & Thal_L \\
\hline \multicolumn{5}{|c|}{ BNST/caudate } \\
\hline $\mathrm{R}$ & 8 & 5 & 2 & BNST_R \\
\hline $\mathrm{L}$ & -10 & 5 & 5 & BNST_L \\
\hline \multicolumn{5}{|c|}{ Basal forebrain } \\
\hline $\mathrm{R}$ & 14 & -1 & -4 & BF_R \\
\hline L & -14 & -1 & -4 & BF_L \\
\hline
\end{tabular}

$L$, Left; $R$, right.

tant to establish that the community assignments (e.g., BNST belongs to a specific community) were representative of the networks at the individual level. First, we compared the community assignments obtained at the group level with those obtained for every individual (based on individual-level data) in terms of Hamming distance (see Results). Second, we used the meta-clustering algorithm (Strehl and Ghosh, 2003), which allowed us to find consensus communities.

Network analysis in general and community detection in particular are complex problems. Although these methods are often applied to larger networks, they are also applicable to smaller ones, as here. A wellestablished problem with community detection is that of resolution limit (Fortunato and Barthélemy, 2007). In many cases, modularity optimization does not subdivide a large number of communities, such that it is not possible to rule out that they are in fact composed of subclusters. Given that our networks are small to start with, this problem is not serious here, but it is possible that the communities detected could be further subdivided, although that was not our goal. Furthermore, as stated below, our aim was to compare network properties as a function of condition. A potential problem with small networks as investigated here is that establishing random networks for comparison purposes may be suboptimal. Critically, however, we do not apply such randomizations to determine the statistical significance of our results, as done in some other work (Lancichinetti et al., 2010). Instead, we use the comparison of network metrics across participants for statistical purposes. In other words, our goal was not to establish that communities are "significant" but to compare parameters obtained from the network decomposition.

\section{Statistical tests}

To perform statistical analysis, modularity, global efficiency, as well as mean edge weight, within community and between community were calculated first at the subject level (community assignment was based on the grouplevel partitions). To determine statistical significance, values calculated at the subject level were compared via the Wilcoxon's signed-rank test, because no assumptions of normality were made on the measures.
Table 2. ROI peak locations for motivation study (Talairach coordinates)

\begin{tabular}{|c|c|c|c|c|}
\hline \multirow[b]{2}{*}{ Location } & \multicolumn{4}{|c|}{ Reward $>$ no-reward } \\
\hline & $x$ & $y$ & $z$ & Label \\
\hline \multicolumn{5}{|l|}{ Parietal } \\
\hline \multicolumn{5}{|c|}{ Intraparietal sulcus } \\
\hline $\mathrm{R}$ & 24 & -54 & 40 & IPS_R \\
\hline L & -27 & -52 & 41 & IPS_L \\
\hline \multicolumn{5}{|c|}{ Inferior parietal lobe } \\
\hline L & -28 & -42 & 41 & IPL_L \\
\hline \multicolumn{5}{|l|}{ Frontal } \\
\hline \multicolumn{5}{|c|}{ Rostral anterior cingulate cortex } \\
\hline $\mathrm{R}$ & 13 & 39 & 8 & rACC_R \\
\hline \multicolumn{5}{|c|}{ Medial prefrontal cortex } \\
\hline $\mathrm{R}$ & 6 & 8 & 39 & MPFC_R \\
\hline L & -8 & 7 & 39 & MPFC_L \\
\hline \multicolumn{5}{|c|}{ Supplementary motor area/pre-SMA } \\
\hline $\mathrm{R} / \mathrm{L}$ & 0 & -6 & 57 & SMA_R \\
\hline \multicolumn{5}{|c|}{ Frontal eye field } \\
\hline $\mathrm{R}$ & 34 & -11 & 48 & FEF_R \\
\hline L & -31 & -12 & 50 & FEF_L \\
\hline \multicolumn{5}{|c|}{ Precentral gyrus } \\
\hline L & -48 & -4 & 37 & PCG_L \\
\hline \multicolumn{5}{|c|}{ Middle frontal gyrus } \\
\hline $\mathrm{R}$ & 26 & 46 & 25 & MFG_R \\
\hline L & -28 & 35 & 29 & MFG_L \\
\hline \multicolumn{5}{|c|}{ Anterior insula } \\
\hline $\mathrm{R}$ & 31 & 17 & 11 & alns_R \\
\hline L & -35 & 26 & 5 & alns_L \\
\hline \multicolumn{5}{|c|}{ Subcortical } \\
\hline \multicolumn{5}{|c|}{ Midbrain } \\
\hline $\mathrm{R}$ & 7 & -15 & -8 & MB_R \\
\hline L & -10 & -18 & -8 & $M B \_L$ \\
\hline \multicolumn{5}{|c|}{ Putamen } \\
\hline $\mathrm{R}$ & 17 & 9 & -2 & Put_R \\
\hline L & -19 & 9 & 2 & Put_L \\
\hline \multicolumn{5}{|c|}{ Caudate } \\
\hline $\mathrm{R}$ & 10 & 9 & 2 & Caud_R \\
\hline $\mathrm{L}$ & -10 & 9 & 2 & Caud_L \\
\hline \multicolumn{5}{|c|}{ Nucleus accumbens } \\
\hline $\mathrm{R}$ & 13 & 6 & -7 & NAcc_R \\
\hline L & -13 & 6 & -7 & NAcc_L \\
\hline
\end{tabular}

L, Left; $R$, right.

We also evaluated changes in the strength of individual connections as a function of context (e.g., threat vs safe). To focus our tests, we compared only connections between communities. This entailed 60 tests in the emotion dataset (see Fig. 5A) and 117 tests in the motivation dataset (see Fig. 8 A). Correction for multiple comparisons used the false discovery rate (FDR) correction method (Benjamini et al., 2006), which is more sensitive when larger numbers of simultaneous tests are performed.

\section{Results}

We applied graph-theoretic analysis to both the threat and reward datasets by considering ROIs as nodes and the trial-by-trial functional connectivity between each pair of ROIs as edge (i.e., connection) strength. Based on the pairwise functional connectivity, the first step was to investigate community structure, namely potential "coherent" groupings of nodes. Community detection is based on the proportion and/or strength of within- to between-community edges, on the intuition that a good community is densely clustered compared with its surroundings (e.g., in the World Wide Web, communities may correspond to groups of pages dealing with the same or related topics). Community detection identifies partitions in networks that maximize specific optimization criteria. They should not be viewed, however, as "true" decompositions of a network, because many possible ways of subdividing the nodes lead to comparable solutions (i.e., val- 
ues close to the maximum) (Good et al., 2010). The central goal of the present study was thus to probe differences in network properties as a function of emotional and motivational stimulus processing. Two main network-level properties (i.e., those that considered all nodes and edges) were computed. The first, global efficiency, provides a measure of integration. A network with higher global efficiency will have "shorter paths." Path distance between two nodes can be defined as the inverse of the functional connectivity between them (the higher the correlation, the shorter the distance). A path between two nodes that involves intermediate nodes is equivalent to the sum of the individual path lengths. The second network-level property, decomposability, indicates how easily a network can be divided in terms of smaller subnetworks (i.e., communities); decreased decomposability reflects less segregation, or equivalently, increased integration, between the different communities. Note that decomposability refers to the network measure called modularity, but we avoid using the latter because of its unintended connotations in cognitive science, such as "encapsulation" (Fodor, 1983). Finally, unlike previous investigations that have focused on analyzing brain networks in terms of binary connectivity graphs, we used methods that operate on weighted graphs that make use of the continuous information of connectivity strength.

\section{Emotion dataset}

Initially, we considered the set of brain regions that exhibited greater responses to threat than to safe cues during the emotion study (Choi et al., 2012). A total of 16 regions were identified (Table 1), including the anterior insula, inferior frontal gyrus, and medial PFC, cortically, and thalamus, BNST/caudate, and basal forebrain, subcortically. These 16 regions comprised the nodes of the network. Trial-by-trial functional connectivity between all pairs of regions during the safe and, separately, threat conditions comprised the edge strength of the safe and threat networks, respectively (see Materials and Methods).

\section{Community structure}

We applied Clauset-Newman-Moore community detection to the set of 16 regions. Community detection was used, separately, during the safe and threat conditions on the group-level connectivity matrix. For both conditions, the network divided into two communities, one comprising cortical regions and another comprising subcortical regions (Fig. 3). Because the partitioning was performed by using the group-level connectivity matrix, they represent a sort of "consensus" decomposition. At the same time, although data at the individual level are noisier, the group-level communities were representative of the communities found at the individual level. Specifically, the median Hamming distance between an individual's community assignment and the group-level community assignment was 1 for the analysis during threat and 1 for the analysis during safe; thus, on average, one node had different assignments when comparing individual networks with the group network. An additional consensus analysis (see Materials and Methods) also converged on the two communities involving subcortical-only and cortical-only regions, with the exception that it placed the right supplementary motor area in a separate community. Inspection of the

\section{Emotion: Safe}

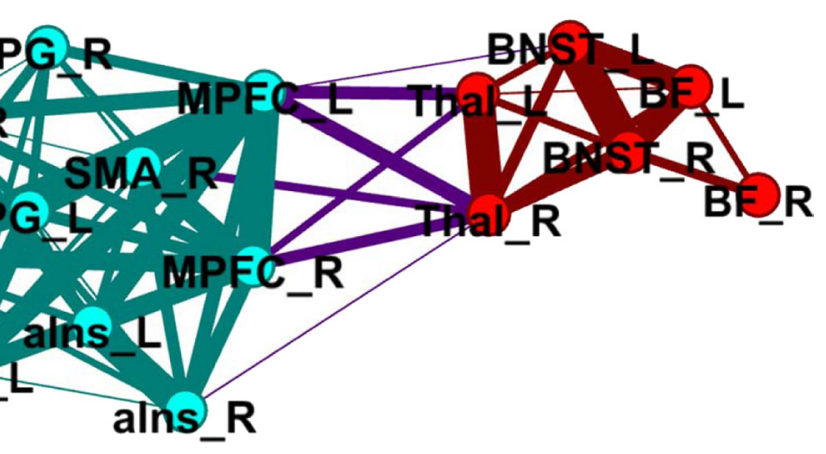

alns_R

Figure 3. Force layout depiction of the group-level network in the emotion dataset during the safe condition. Nodes are colored to show community organization (red for subcortical community, teal for cortical community). Edges are also colored according to communities with between-community edges colored purple. For visualization purposes, edges were thresholded to leave only the $\sim 50 \%$ strongest ones. Visualization done with Gephi 0.8 beta, making use of the Force Atlas and label-adjust layout tools. For

Table 3. Network- and community-level measures

\begin{tabular}{llc}
\hline Network measure & Threat-safe & Reward-control \\
\hline Global efficiency & $\uparrow(p=\mathbf{0 . 0 0 5 4 )}$ & $\uparrow(p=\mathbf{0 . 0 0 0 5 )}$ \\
Decomposability (modularity Q) & $\downarrow(p=\mathbf{0 . 0 0 1 9 )}$ & $\downarrow(p=\mathbf{0 . 0 0 0 2 )}$ \\
Mean edge weight within subcortical & $\uparrow(p=\mathbf{0 . 0 3 4 1 )}$ & NS $(p=0.1080)$ \\
Mean edge weight within cortical & NS $(p=0.4800)$ & $\uparrow(p=6.67 e-4)$ \\
Mean edge weight between communities & $\uparrow(p=4.5620$ e-4) & $\uparrow(p=7.16 e-4)$ \\
\hline
\end{tabular}

Arrows denote the direction of change, and items in bold are statistically significant. NS, Nonsignificant.

individual-level networks revealed that this result was primarily driven by two participants who had three instead of two detected communities, with the "extra" community involving the right supplementary motor area; constraining the consensus algorithm to detect only two communities revealed the assignments described above. Based on these results, we used the group-level community assignment in the subsequent analyses.

We also evaluated the quality of the community structure. This can be accomplished by comparing the decomposability value obtained for a network with the corresponding decomposability of a random graph of the same size and expected degree distribution (see Materials and Methods). The comparison is typically expressed as a $z$-score, and the values observed were 12.4 for safe and 10.6 for threat, indicating strong community structure in both cases (i.e., $z$ much larger than 2; for a comment on small networks, see Materials and Methods).

\section{Network- and community-level measures}

Network- and community-level measures were determined during safe and threat contexts and contrasted to each other (Table 3). To allow a statistical comparison of the results, measures were determined at the individual level and contrasted across conditions. The composition (i.e., nodes) of the two communities was the one determined at the group-level network, but the connectivity matrix was based on each individual's data.

Global efficiency increased and decomposability decreased during threat. We evaluated how mean connection weights changed within and between communities as a function of threat (i.e., threat vs safe). To do so, changes in connection strength were considered at the individual level and compared across the group (via a Wilcoxon's signed-rank test). Mean connection weights both between communities and within subcortical community increased during threat (Table 3 ). However, in the case of 


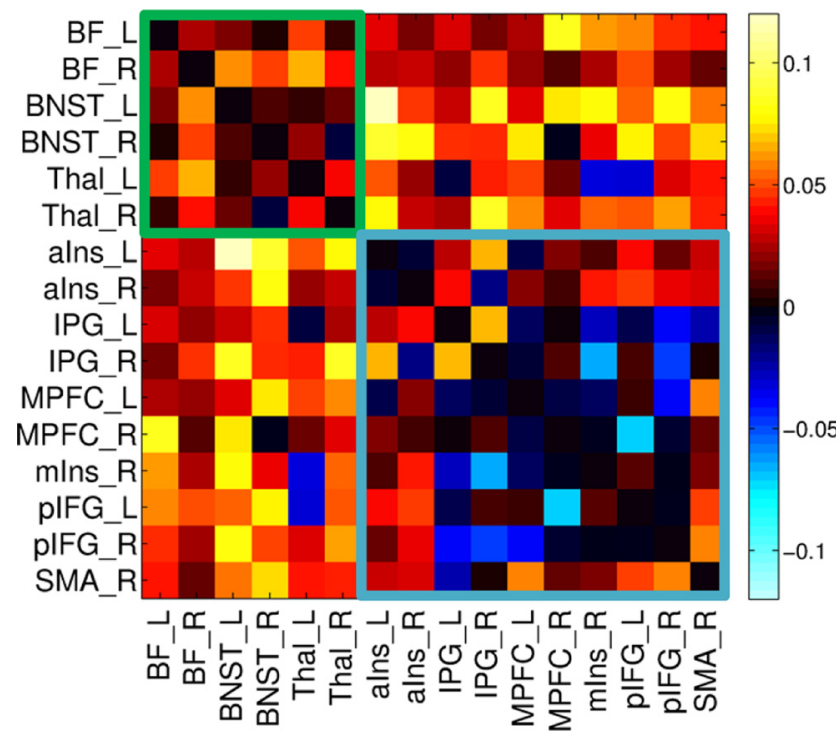

Figure 4. Changes in threat versus safe connectivity for the group-level network in the emotion dataset. Within-cortical connections are bounded by a teal box, and within-subcortical connections are bounded by a green box. For abbreviations, see Table 1.

the cortical community, a significant change of connection strength was not observed; in fact, some connection decreases were observed (Fig. 4).

Finally, we probed changes in the strength of individual connections as a function of context. We were particularly interested in considering connections between the two communities. When corrected for multiple comparisons (via FDR), increases in connection strength were detected as the context changed from safe to threat (Fig. $5 A, B$ ). The polar plots shown in Figure $5 C$ highlight some of the changes. For instance, the right anterior insula exhibited significant increases to all subcortical regions but one, with the largest increases to the right/left BNST, and then to the right thalamus. Also shown are the changes for the right BNST and right thalamus.

\section{Control analyses}

For the analyses above, ROIs were selected based on the contrast threat $>$ safe. This selection criterion does not bias the results reported above for the following reasons. ROIs were selected based on the "average" response to threat and safe conditions, whereas the network analysis used trial-by-trial estimates, which are in fact independent of average responses. Note also that, when estimating the average responses to the threat and safe conditions based on standard multiple regression (as done here), the trialby-trial fluctuations around the mean are treated as error. Thus, an increase in the overall correlations is not necessitated by the selection criterion.

In any case, we formally tested for any influence of the selection criterion on the observed results. To do so, we evaluated whether average increases in responses were correlated with increases in correlated responses between regions based on trial-by-trial data. Specifically, for each subject, we defined two variables: abs $\left[\left(X_{\text {threat }}-\right.\right.$ $\left.X_{\text {safe }}\right)-\left(Y_{\text {threat }}-Y_{\text {safe }}\right)$, which indicates the difference in average signal increase between the safe and threat conditions for regions $X$ and $Y$; and abs $\left[r(X, Y)_{\text {threat }}-r(X, Y)_{\text {safe }}\right]$, which indicates the difference in correlation between regions $X$ and $Y$ when changing from safe to threat based on trial-by-trial estimates (in both cases, absolute values were considered because direction was not important, only magnitude). After Fisher's $z$ transforming, we tested whether these correlations were significantly different from zero across participants. The mean correlation was 0.0184 (before transforming), and the values did not differ significantly from zero $(p=0.3958)$. Thus, increases in average differential responses in the emotion dataset did not generate increases in functional connectivity between regions.

Another potential concern was that some of the present findings were driven by distance between ROIs. Our goal was not to understand connectivity during specific conditions but differences in connectivity between conditions. Thus, spatial proximity would not be expected to have an impact on these differential relationships. Nevertheless, to test the question formally, we correlated the Euclidean distance between ROIs and the differential connectivity (safe vs threat) for each pair of ROIs. The mean robust correlation (across subjects) was negligible $(r=-0.05$, NS). Furthermore, when we ran the community-detection algorithm on Euclidean distances between regions, the communities detected did not bear any semblance to the ones reported.

\section{Motivation dataset}

Similar to the analysis above, we considered the set of brain regions that exhibited greater responses to reward than to control cues during the motivation study (Padmala and Pessoa, 2011). A total of 22 regions were identified (Table 2), including the anterior insula, medial PFC, and middle frontal gyrus, cortically, and midbrain, caudate, putamen, and nucleus accumbens, subcortically. These 22 regions comprised the nodes of the network. Trialby-trial functional connectivity between all pairs of regions during the control and, separately, reward conditions comprised the edge weights of the control and reward networks, respectively (see Materials and Methods).

\section{Community structure}

Community detection was used, separately, during the reward and control conditions. For both conditions, the network divided into two communities, one comprised entirely cortical regions and another comprised subcortical regions and the right rostral anterior cingulate cortex (ACC) (Fig. 6).

Again, to evaluate the reliability of the community assignments, we determined the median Hamming distance between an individual's community assignment and the group-level community assignment, which revealed a value of 2.5 for the analysis during reward and a value of 2 for the analysis during control, so on average, 2.5 and 2 nodes had different assignments when comparing individuals with the group network. Thus, although data at the individual level are noisier, the group-level communities were representative of the findings at the individual level. An additional consensus analysis also converged on the communities identified above. Based on these results, we used the grouplevel community assignments in the subsequent analyses.

As done previously, the quality of the community structure was determined by comparing the observed decomposability with the corresponding decomposability of a random graph of the same size and expected degree distribution (see Materials and Methods). The $z$-score values observed were 13.0 for control and 13.2 for reward, indicating strong community structure (i.e., $z$ much larger than 2; for a comment on small networks, see Materials and Methods).

\section{Network- and community-level measures}

Community-level measures were determined during control and reward contexts and contrasted to each other. Several of the findings when considering the change from control to reward contexts paralleled those observed when the changes involved safe 
A

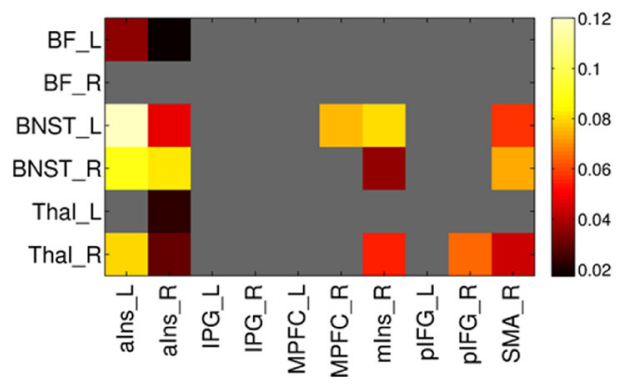

B Emotion: Significant changes between communities

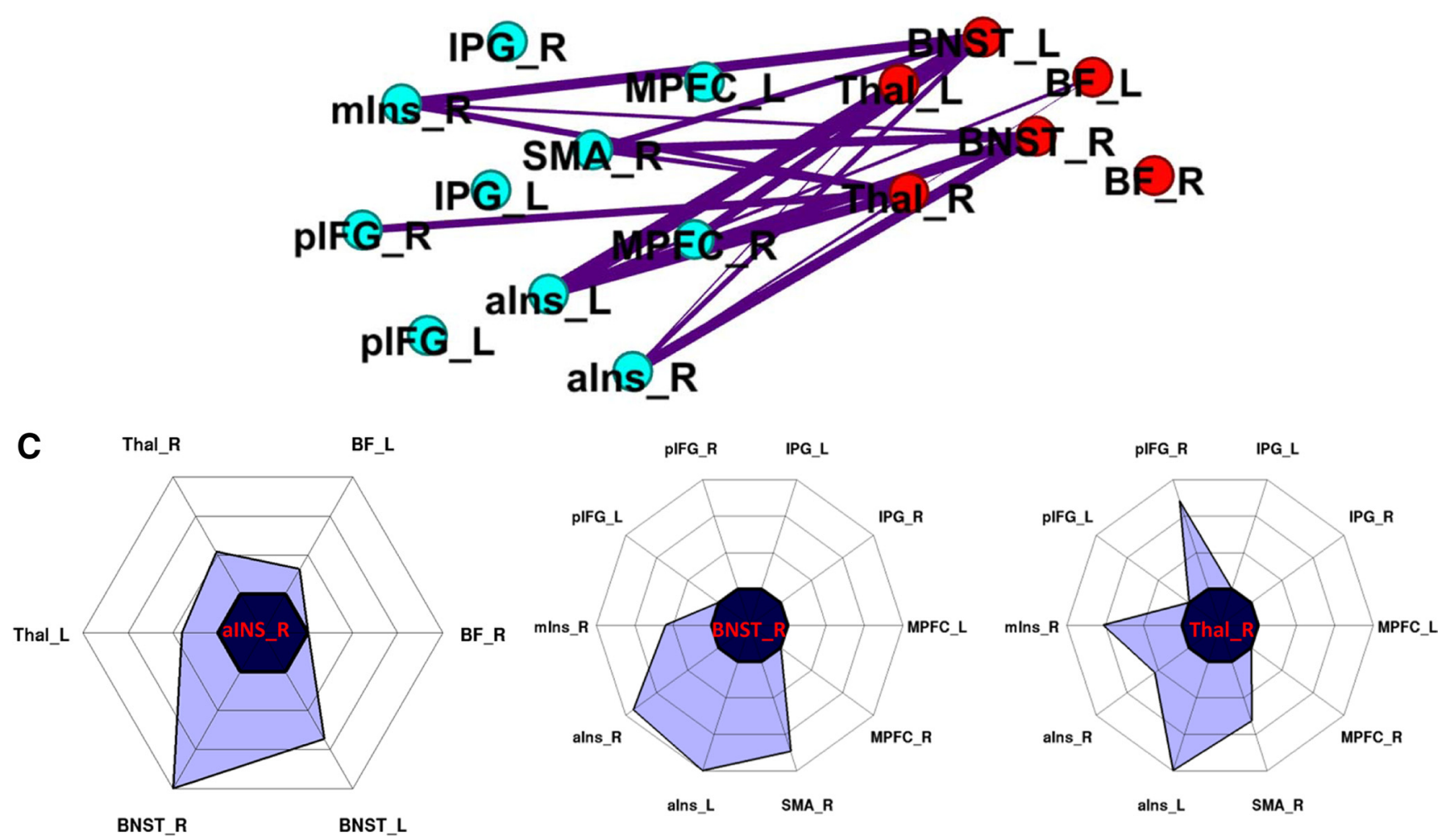

Figure 5. A, Changes in threat versus safe connectivity between communities for the group-level network in the emotion dataset. Only significant ( $p<0.05$, FDR corrected) changes are shown (nonsignificant connections are gray). $\boldsymbol{B}$, Only between-community edges with significant threat versus safe connectivity changes are shown ( $p<0.05$, FDR corrected). Node positions and colors as in Figure 3. Visualization done with Gephi 0.8 beta, making use of the Force Atlas and label-adjust layout tools. C, Polar plots illustrating the changes of key cortical and subcortical nodes from $\boldsymbol{A}$. Distance from the dark centered hexagon/decagon corresponds to change in connectivity (threat-safe). Nonsignificant changes were set to zero. For abbreviations, see Table 1.

and threat. Global efficiency increased and decomposability decreased during reward. We also evaluated how mean connection (i.e., edge) weights between and within communities changed during reward (relative to control) (Fig. 7). Mean connection weights increased between communities and within the cortical community (Table 3). No significant difference was observed within the subcortical community.

We also probed changes in the strength of individual connections between the subcortical and cortical communities as a function of context. As shown in Figure 8, $A$ and $B$, many connections increased during reward (vs control) and survived multiple comparisons. The polar plots show significant changes of the left/ right caudate and right nucleus accumbens (Fig. 8C).

\section{Control analyses}

The same control analysis as in the emotion study was performed here. Again, we evaluated whether average increases in responses were correlated with increases in correlated responses between re- gions based on the trial-by-trial data. Specifically, for each subject, we defined two variables: $\operatorname{abs}\left[\left(X_{\text {reward }}-X_{\text {control }}\right)-\left(Y_{\text {reward }}-\right.\right.$ $\left.Y_{\text {control }}\right)$, which indicates the difference in average signal increase between the reward and control conditions for regions $X$ and $Y$; and $\operatorname{abs}\left[r(X, Y)_{\text {reward }}-r(X, Y)_{\text {control }}\right]$, which indicates the difference in correlation between regions $X$ and $Y$ when changing from control to reward based on trial-by-trial estimates. After Fisher's $z$ transforming, we tested whether these correlations were significantly different from zero across participants. The mean correlation was 0.0017 (before transforming), and the values did not differ significantly from zero $(p=0.9069)$. Thus, increases in average differential responses in the motivation dataset did not generate increases in functional connectivity between regions.

To test for the impact of spatial distance, we correlated the Euclidean distance between ROIs and the differential connectivity (control vs reward) for each pair of ROIs. The mean robust correlation (across subjects) was negligible $(r=0.10$, NS). When 


\section{Motivation: Control}

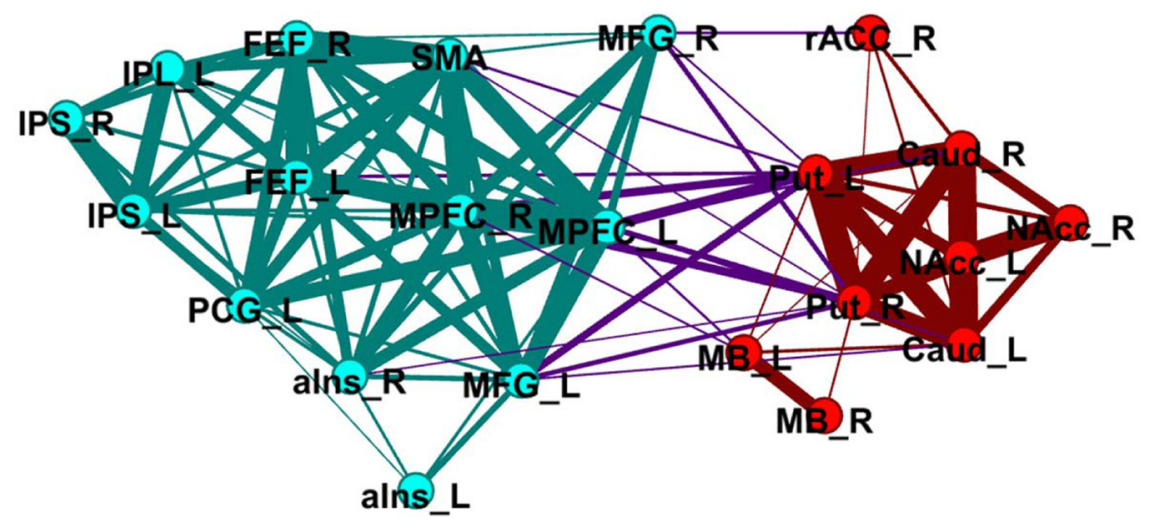

Figure 6. Force layout depiction of the group-level network in the motivation dataset during the control condition. Nodes are colored to show community organization (red for subcortical community, teal for cortical community). Edges are also colored according to communities with between-community edges colored purple. For visualization purposes, edges were thresholded to leave only the $\sim 40 \%$ strongest ones. Visualization done with Gephi 0.8 beta, making use of the Force Atlas and label adjust-layout tools. For abbreviations, see Table 2.

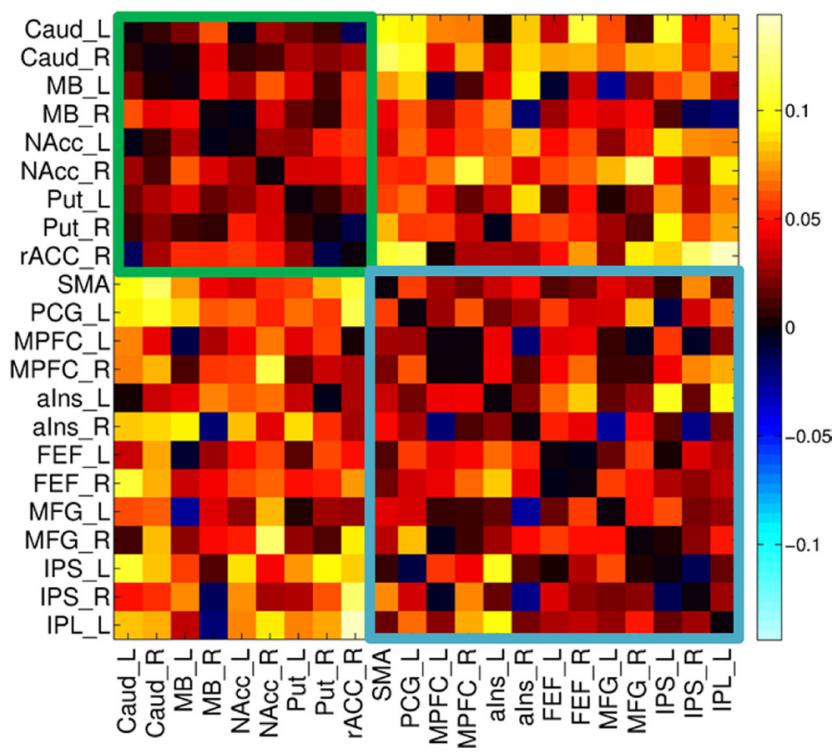

Figure 7. Changes in reward versus control connectivity for the group-level network in the motivation dataset. Within-cortical connections are bounded by a teal box, and withinsubcortical edges are bounded by a green box. For abbreviations, see Table 2 .

we ran the community-detection algorithm on Euclidean distances between regions, different communities were detected relative to the ones reported for the motivation dataset.

\section{Discussion}

Graph-theoretic network analysis was used to characterize how emotional and motivational mini-states potentially alter functional connectivity between brain regions. Both threat and reward caused significant changes in network- and communitylevel measures, as discussed next.

\section{Emotion dataset}

The emotional manipulation involved the threat of shock, which could occur during the delay interval after the cue stimulus. We only analyzed trials that did not contain physical shock because our goal was to understand how shock anticipation/monitoring affected brain function. The effectiveness of the manipulation was confirmed by the observations that skin conductance responses increased after threat cues and that, behaviorally, response interference increased during threat trials (Choi et al., 2012).

Community detection applied to regions responsive to threat monitoring identified two subnetworks, one comprising cortical regions and another comprising subcortical regions. As stated, communities should not be viewed as "true" decompositions of a network. Real-world networks have a large number of partitions whose decomposability (i.e., graph modularity) values are close to the global maximum (Fortunato, 2010). The central goal of the present study was thus to probe differences in network properties as a function of emotional and motivational stimulus processing. During threat, the tendency of the overall network to form two separate communities, that is, decomposability, decreased. In other words, the two communities became less clearly defined during the threat condition. In a related manner, global efficiency increased during threat. Global efficiency is a measure of integration and provides one way to estimate the potential for functional integration between brain regions. As suggested by Achard and Bullmore (2007), it provides a measure of the capacity of the network for information transfer between nodes. Together, these results suggest that responses generated by a given region were capable of affecting another region more effectively during threat.

It is relevant to discuss the findings regarding the BNST given the importance of this region in anxiety-related mechanisms (Davis et al., 2010; Somerville et al., 2010). During threat, the BNST increased functional connectivity with both anterior and mid-insula, as well as regions of dorsomedial PFC (Fig. 5). In contrast, the amygdala, which is engaged during the processing of more punctate conditioning stimuli used in aversive conditioning studies (Büchel et al., 1998; LaBar et al., 1998; Lim et al., 2009), did not exhibit enhanced response during threat cues (vs safe) and thus was not included in the network analysis (for additional discussion concerning the amygdala, see Choi et al., 2012). The thalamus, another region implicated in threat processing (Kalin et al., 2005), also exhibited increased functional connectivity with both anterior and mid-insula regions. Together, these results indicate that an important aspect of threat processing involves enhanced functional integration between subcortical regions, such as the BNST and thalamus, and cortical regions, including the insula and medial PFC. These findings were observed when performance was irrelevant to the administration of the negative stimulus (i.e., shock), unlike the situation in the reward manipulation discussed next.

\section{Motivation dataset}

As in the case of emotion, motivation was manipulated via an initial cue stimulus, which in this case signaled the possibility of obtaining a reward given correct and fast task performance. The effectiveness of the motivation manipulation was attested by the pattern of behavioral performance during the response-conflict task: subjects were faster and exhibited less response interference during reward trials (Padmala and Pessoa, 2011). 


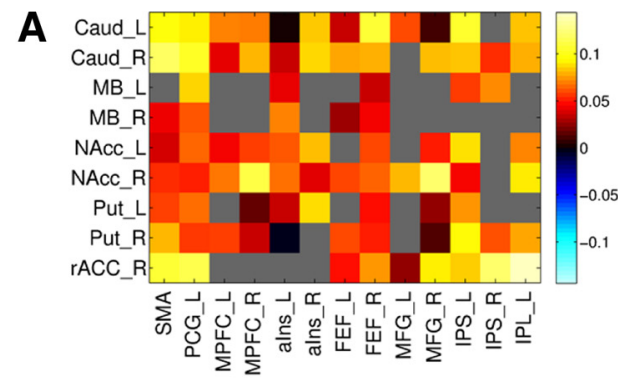

\section{B}

\section{Motivation: Significant changes between communities}

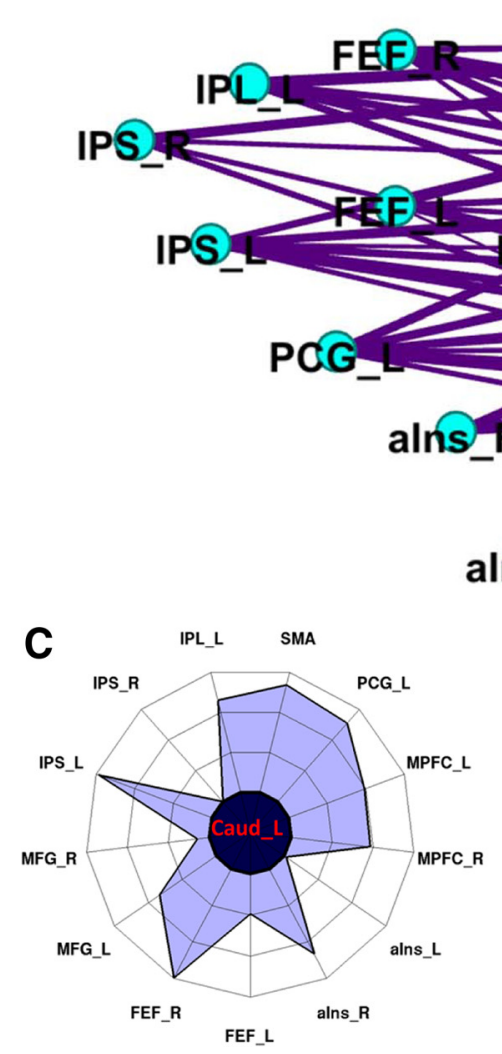

Figure 8. $A$, Change in reward versus control connectivity between communities for the group-level network in the motivation dataset. Only significant $(p<0.05$, FDR corrected) changes are shown (nonsignificant connections are gray). $\boldsymbol{B}$, Only between-community edges with significant reward versus control connectivity changes are shown ( $p<0.05$, FDR corrected). Node positions and colors as in Figure 6. Visualization done with Gephi 0.8 beta, making use of the Force Atlas and label-adjust layout tools. C, Polar plots illustrating three key subcortical nodes from $A$. Distance from the dark centered polygon corresponds to change in connectivity (reward- control). Nonsignificant changes were set to zero. For abbreviations, see Table 2.

Community detection applied to regions that responded more strongly to reward versus control cues identified two partitions, one comprising cortical regions and another comprising subcortical regions in addition to the right rostral ACC. As in the case of threat, global efficiency increased and decomposability decreased with reward. Furthermore, average between-community connection increased with reward. The involvement of the nucleus accumbens and the caudate in reward-related processing is well documented (Haber and Knutson, 2010). In our paradigm, these regions consistently increased their functional connectivity with cortical regions.

\section{Comparing emotion and motivation datasets}

The effects of emotional and motivational cues exhibited several similarities. For example, increased functional connectivity was observed between communities in both cases. At the network level, global efficiency increased and decomposability decreased. Thus, the communities became less segregated with the context signaled by the cue (potential shock or reward), revealing that one way in which emotional and motivational processing affect brain responses is by increasing functional connections across brain regions.

A notable difference between the results of the two datasets concerns how functional connectivity changed within the two communities. In the case of reward, functional connectivity increased in the cortex but was not robustly altered within the subcortical community. In the case of threat, functional connectivity did not change within cortex but increased within the subcortical community. In fact, in cortex, several changes in connection strength were numerically negative (i.e., they reduced with threat). How should we interpret these findings?

Let us consider the motivation dataset first. We have proposed previously that the effect of reward during perception and cognition depends on interactions between valuation regions and frontoparietal regions important for attention and executive con- 
trol (Pessoa and Engelmann, 2010). Such interactions lead to the upregulation of control and improved behavioral performance during challenging task conditions (and higher likelihood of reward). Along these lines, Harsay et al. (2011) reported higher functional connectivity between the caudate and both the frontal eye field and intraparietal sulcus during the reward condition of an antisaccade task. In the context of our study, the increased functional connectivity between the two communities in the motivation dataset is consistent with these ideas, and they also suggest that the increases in connectivity can be quite broad. For example, the left/right caudate and the right nucleus accumbens exhibited increases in functional connectivity to all cortical regions but one.

In terms of the emotion dataset, we and others have proposed that emotional processing diverts processing resources that are also needed for executive function. This interference effect is then proposed to impair cognitive performance (Pessoa, 2009). We tentatively suggest that the reduced functional connectivity among some of the cortical areas may have reflected the interference effect that the threat of shock exerted on subsequent cognitive performance: it is possible that responses across some of the cortical regions became less coherent with threat. It is noteworthy that the left/right anterior insula did not exhibit the same pattern of reduced functional connectivity to other cortical regions. During threat, not only did these regions increase their correlation with regions in the subcortical community but to regions in the cortical community, too. These findings are consistent with the notion that the anterior insula plays a distinctive role during threat monitoring (Paulus and Stein, 2006). It also should be noted that the threat and reward manipulations were asymmetric, such that threat was independent of performance, whereas reward was contingent on it. Therefore, the differences discussed here may have partly reflected this distinction.

It is conceivable that any type of comparison between a more challenging or interesting condition and a less challenging/interesting one would produce some of the results observed here. To investigate this issue, we repeated our analyses (in the emotion dataset), but this time using the trial-based target phase responses from regions that responded more vigorously to incongruent versus neutral trials. No significant changes in network- or community-level measures were detected between incongruent and neutral conditions (all $p$ values $>0.15$; tests as in Table 3 ). It should be noted, however, that a recent MEG study reported that greater cognitive effort was associated with the emergence of a more efficient and less modular network topology (Kitzbichler et al., 2011), indicating that this type of change is not specific to the emotional and motivational manipulations we probed. Finally, additional studies are needed to determine how our results generalize to emotional and motivational processing in different task contexts beyond the one analyzed here.

\section{Conclusions}

Many studies have investigated large-scale network properties of the brain, typically during the resting state (Wang et al., 2010). These studies have used a large number of regions, ranging from $\sim 100$ to $>1000$. In the current study, we pursued a different approach. We focused on a small number of regions $(\sim 20)$ spanning cortical and subcortical sites that were robustly engaged by emotional and motivational manipulations. Our findings revealed a number of important ways in which both emotional and motivational processing altered functional connectivity, including increased global efficiency and reduced decomposability. As mentioned, a recent MEG study has suggested that greater cog- nitive effort is associated with the emergence of a less modular network topology (Kitzbichler et al., 2011). Given that MEG was used, it is likely that the changes observed were more directly tied to cortical processing. The present findings revealed that the processing of emotional and motivational stimuli may have a similar impact on network organization but emphasize enhanced vertical, cortical-subcortical functional integration in a manner that may be behaviorally appropriate. Potential reward may thus contribute to improved task performance (and reward attainment), and potential threat may redirect mental resources in the service of mobilizing the body toward safeguarding the organism against harm.

\section{References}

Aarts E, van Holstein M, Cools R (2011) Striatal dopamine and the interface between motivation and cognition. Front Psychol 2:163.

Achard S, Bullmore E (2007) Efficiency and cost of economical brain functional networks. PLoS Comput Biol 3:e17.

Benjamini Y, Krieger AM, Yekutieli D (2006) Adaptive linear step-up procedures that control the false discovery rate. Biometrika 93:491-507.

Büchel C, Morris J, Dolan RJ, Friston KJ (1998) Brain systems mediating aversive conditioning: an event-related fMRI study. Neuron 20:947-957.

Bullmore E, Sporns O (2009) Complex brain networks: graph theoretical analysis of structural and functional systems. Nat Rev Neurosci 10:186-198.

Chandrasekhar PV, Capra CM, Moore S, Noussair C, Berns GS (2008) Neurobiological regret and rejoice functions for aversive outcomes. Neuroimage 39:1472-1484.

Choi JM, Padmala S, Pessoa L (2012) Impact of state anxiety on the interaction between threat monitoring and cognition. Neuroimage 59:1912-1923.

Clauset A, Newman ME, Moore C (2004) Finding community structure in very large networks. Phys Rev E Stat Nonlin Soft Matter Phys 70:066111.

Cohen MS (1997) Parametric analysis of fMRI data using linear systems methods. Neuroimage 6:93-103.

Cox RW (1996) AFNI: software for analysis and visualization of functional magnetic resonance neuroimages. Comput Biomed Res 29:162-173.

Dalton KM, Kalin NH, Grist TM, Davidson RJ (2005) Neural-cardiac coupling in threat-evoked anxiety. J Cogn Neurosci 17:969-980.

Davis M, Walker DL, Miles L, Grillon C (2010) Phasic vs sustained fear in rats and humans: role of the extended amygdala in fear vs anxiety. Neuropsychopharmacology 35:105-135.

Fodor JA (1983) The modularity of mind: an essay on faculty. Psychology. Cambridge, MA: Massachusetts Institute of Technology.

Fortunato S (2010) Community detection in graphs. Phys Rep 486:75-174.

Fortunato S, Barthélemy M (2007) Resolution limit in community detection. Proc Natl Acad Sci U S A 104:36-41.

Friston KJ, Buechel C, Fink GR, Morris J, Rolls E, Dolan RJ (1997) Psychophysiological and modulatory interactions in neuroimaging. Neuroimage 6:218-229.

Good BH, de Montjoye YA, Clauset A (2010) Performance of modularity maximization in practical contexts. Phys Rev E Stat Nonlin Soft Matter Phys 81:046106.

Haber SN, Knutson B (2010) The reward circuit: linking primate anatomy and human imaging. Neuropsychopharmacology 35:4-26.

Harsay HA, Cohen MX, Oosterhof NN, Forstmann BU, Mars RB, Ridderinkhof KR (2011) Functional connectivity of the striatum links motivation to action control in humans. J Neurosci 31:10701-10711.

Kalin NH, Shelton SE, Fox AS, Oakes TR, Davidson RJ (2005) Brain regions associated with the expression and contextual regulation of anxiety in primates. Biol Psychiatry 58:796-804.

Kitzbichler MG, Henson RN, Smith ML, Nathan PJ, Bullmore ET (2011) Cognitive effort drives workspace configuration of human brain functional networks. J Neurosci 31:8259-8270.

LaBar KS, Gatenby JC, Gore JC, LeDoux JE, Phelps EA (1998) Human amygdala activation during conditioned fear acquisition and extinction: a mixed-trial fMRI study. Neuron 20:937-945.

Lancichinetti A, Radicchi F, Ramasco JJ (2010) Statistical significance of communities in networks. Phys Rev E Stat Nonlin Soft Matter Phys 81:046110.

Lim SL, Padmala S, Pessoa L (2009) Segregating the significant from the 
mundane on a moment-to-moment basis via direct and indirect amygdala contributions. Proc Natl Acad Sci U S A 106:16841-16846.

Mobbs D, Yu R, Rowe JB, Eich H, FeldmanHall O, Dalgleish T (2010) Neural activity associated with monitoring the oscillating threat value of a tarantula. Proc Natl Acad Sci U S A 107:20582-20586.

Newman ME (2010) Networks: an introduction. New York: Oxford UP.

Padmala S, Pessoa L (2011) Reward reduces conflict by enhancing attentional control and biasing visual cortical processing. J Cogn Neurosci 23:3419-3432.

Paulus MP, Stein MB (2006) An insular view of anxiety. Biol Psychiatry 60:383-387.

Pessoa L (2009) How do emotion and motivation direct executive function? Trends Cogn Sci 13:160-166.

Pessoa L, McKenna M, Gutierrez E, Ungerleider LG (2002) Neural processing of emotional faces requires attention. Proc Natl Acad Sci U S A 99:11458-11463.

Pessoa L, Engelmann JB (2010) Embedding reward signals into perception and cognition. Front Neurosci 4.pii:17.

Rissman J, Gazzaley A, D’Esposito M (2004) Measuring functional connectivity during distinct stages of a cognitive task. Neuroimage 23:752-763.

Rubinov M, Sporns O (2010) Complex network measures of brain connectivity: uses and interpretations. Neuroimage 52:1059-1069.

Rubinov M, Sporns O (2011) Weight-conserving characterization of complex functional brain networks. Neuroimage 56:2068-2079.
Savine AC, Braver TS (2010) Motivated cognitive control: reward incentives modulate preparatory neural activity during task-switching. J Neurosci 30:10294-10305.

Schwarz AJ, McGonigle J (2011) Negative edges and soft thresholding in complex network analysis of resting state functional connectivity data. Neuroimage 55:1132-1146.

Somerville LH, Whalen PJ, Kelley WM (2010) Human bed nucleus of the stria terminalis indexes hypervigilant threat monitoring. Biol Psychiatry $68: 416-424$.

Street JO, Carroll RJ, Ruppert D (1988) A note on computing robust regression estimates via iteratively reweighted least squares. Am Stat 42:152-154

Strehl A, Ghosh J (2003) Cluster ensembles-a knowledge reuse framework for combining multiple partitions. J Mach Learn Res 3:583-617.

Wang J, Zuo X, He Y (2010) Graph-based network analysis of resting-state functional MRI. Front Syst Neurosci 4:16.

White S, Smyth P (2005) A spectral clustering approach to finding communities in graphs. Paper presented at the Fifth SIAM International Conference on Data Mining, Newport Beach, CA, April.

Zachary WW (1977) An information flow model for conflict and fission in small groups. J Anthropol Res 33:452-473.

Zhou D, Thompson WK, Siegle G (2009) MATLAB toolbox for functional connectivity. Neuroimage 47:1590-1607. 